\title{
Construction and Case Study of Flexibility Evaluation System for Power Generation Enterprises in the Auxiliary Service Market
}

\author{
Ying Le ${ }^{1}$, Baozhong Zhou ${ }^{1}$, Wenbin Zhu ${ }^{1}$ \\ ${ }^{1}$ Huadian Electric Power Research Institute Co., Ltd., Hangzhou, China
}

\begin{abstract}
At present, the flexible transformation of thermal power is an effective measure to solve the problem of renewable energy consumption. At the same time, the improvement of the electricity auxiliary service market makes the power generation enterprises improve their ability to provide auxiliary services through flexible transformation and obtain benefits while serving the power system. Therefore, how to construct the flexibility evaluation system of regional units according to the characteristics of the auxiliary service market in different regions is of great significance to power generation enterprises.In this paper, the flexibility evaluation system of the auxiliary market environment is designed. Analytic hierarchy process and fuzzy comprehensive evaluation method were used to determine the weight and final evaluation basis.
\end{abstract}

\section{The introduction}

The "13th Five-Year Plan" period is a key stage in the transformation of China's energy consumption structure. As one of the main measures of the power supply side reform, the flexibility transformation of thermal power has been highly valued by the energy authorities. With the further deepening of the power industry system reform, it is necessary and urgent to study the power market, especially the auxiliary services. Auxiliary service is an important feature of the power market, which has a direct impact on the safety, quality and economy of power system operation.

Recently, the market mechanism for electricity auxiliary services has been launched in 14 regions of the country. In light of the actual situation, a system of basic market rules has been established in these regions. The results have been shown in promoting the safe and stable operation of power systems, promoting the elimination of renewable energy sources, improving system peak modulation capabilities and equipment utilization efficiency, and promoting the development of new technologies and equipment.

At the same time, the market main body to the electric power auxiliary service market awareness has been improved. The power generation company provides auxiliary services from passive to active, improves its ability to provide auxiliary services through flexible transformation, and gains revenue while serving the power system. It has changed from "I want to provide" to "I want to provide".

Various localities have also successively issued the "two rules" document, which stipulates the rules for compensation, assessment and compensation, and costsharing of power auxiliary services. The planned compensation method stipulated in the "Two Rules" can to some extent motivate the generating units to provide power auxiliary services.Due to different market policies for auxiliary services in various regions, there are differences in equipment of power generation enterprises and there is a lack of methods to solve flexibility evaluation problems based on equipment differences under different market policies for auxiliary services. Power generation enterprises often use the form of empirical judgment to distinguish the level of flexibility, which can easily lead to insufficient consideration of factors and inaccurate judgment results, which directly affects the competitiveness of power generation enterprises in the auxiliary service market. In view of this, this paper constructs the flexibility evaluation system of power generation enterprises and determines the evaluation method in order to play a practical evaluation role in power generation enterprises.

\section{Construction of Flexibility Evaluation Index for Power Generation Enterprises}

Power generation enterprises involving multi-source power usually cover thermal power, hydropower, wind power, photovoltaic and other power generation systems. This paper only aims at the thermal power plant and constructs the flexible evaluation index system.In the auxiliary services market environment, flexibility evaluation factors mainly include (1)Policy.The policy orientation of the region is related to the level of flexibility of generating units in the region, reflecting the competitiveness of power generation enterprises in the region's electricity service market. It mainly includes the types of transaction of auxiliary services, the market price of auxiliary services, the triggering conditions of auxiliary services, the limited conditions for the unit to participate in auxiliary services, and the market technical

*ying-le@chder.com 
indicators for the unit to participate in auxiliary services. (2) Flexibility indicators. The flexibility index directly reflects the performance of the unit and the ability of the generator set to participate in the power auxiliary service under the regional policy.It mainly includes Load following rate, adjustment rate, adjustment accuracy, response time, peak range, cold reserve, time used for grid-connected, climbing rate, etc. (3) Market demand. Market demand directly affects the types of auxiliary services that power generation enterprises participate in, and affects the economic benefits of power generation enterprises, including FM, peak adjustment, reactive adjustment, backup, and black start.(4) Cost. Cost mainly reflects the cost competitiveness of external products of power generation companies, including electricity, auxiliary service revenue, coal consumption, and environmental protection costs.

Based on the policy, flexibility index, market demand and cost as a starting point. In the construction of indicators, follow the principles of scientific, comparable, growing, systematic, and quantifiable design to obtain objective and accurate evaluation results.

Based on the above principles, the flexibility evaluation was finalized from four aspects: regional policy, market demand, flexibility indicators, and cost, after conducting a survey of specific power generation companies and soliciting the opinions of many experts in the industry, as shown in table 1 .

Table 1. Total factor competitiveness evaluation index.

\begin{tabular}{|c|c|c|}
\hline NO. & Index 1 & Index 2 \\
\hline 1 & \multirow{3}{*}{ Policy (B1) } & $\begin{array}{l}\text { Type of transaction } \\
\text { (C11) }\end{array}$ \\
\hline 2 & & Market price (C12) \\
\hline 3 & & $\begin{array}{l}\text { Trigger condition } \\
\text { (C13) }\end{array}$ \\
\hline 4 & \multirow{5}{*}{$\begin{array}{l}\text { Market } \\
\text { demand } \\
\text { (B2) }\end{array}$} & $\begin{array}{c}\text { frequency } \\
\text { modulation }(\mathrm{C} 21)\end{array}$ \\
\hline 5 & & Peak adjustment (C22) \\
\hline 6 & & $\begin{array}{l}\text { Reactive power } \\
\text { regulation (C23) }\end{array}$ \\
\hline 7 & & standby (C24) \\
\hline 8 & & Black boot (C25) \\
\hline 9 & \multirow{8}{*}{$\begin{array}{c}\text { Flexibility } \\
\text { indicators } \\
\text { (B3) }\end{array}$} & $\begin{array}{l}\text { load following rate } \\
\text { (C31) }\end{array}$ \\
\hline 10 & & adjustment rate $(\mathrm{C} 32)$ \\
\hline 11 & & $\begin{array}{c}\text { adjustment accuracy } \\
\text { (C33) }\end{array}$ \\
\hline 12 & & response time $(\mathrm{C} 34)$ \\
\hline 13 & & peak range (C35) \\
\hline 14 & & cold reserve (C36) \\
\hline 15 & & $\begin{array}{l}\text { time used for grid- } \\
\text { connectedadjust } \\
\text { (C37) }\end{array}$ \\
\hline 16 & & climbing rate (C38) \\
\hline 17 & \multirow{2}{*}{ Cost (B4) } & electricity (C41) \\
\hline 18 & & $\begin{array}{l}\text { auxiliary service } \\
\text { revenue }(\mathrm{C} 42)\end{array}$ \\
\hline
\end{tabular}

\begin{tabular}{|c|c|}
\hline 19 & $\begin{array}{c}\text { coal consumption } \\
\text { (C43) }\end{array}$ \\
\hline \multirow{2}{*}{20} & $\begin{array}{c}\text { environmental } \\
\text { protection costs } \\
\text { (C44) }\end{array}$ \\
\hline
\end{tabular}

\section{Index weighting and flexibility evaluation methodology}

According to the above index system, it is necessary to establish a scientific evaluation model and method to evaluate the flexibility of power generation enterprises. Through the evaluation, the real situation of the enterprise can be reflected truthfully. This paper adopts fuzzy comprehensive evaluation method, which is a widely used method. It has the characteristics of clear results and strong system. It can solve fuzzy and difficult to quantify problems and is suitable for solving various non-deterministic problems. Based on the flexibility index system, a flexibility evaluation model for power generation enterprises is constructed.

\subsection{Determination of index weight}

Whether the weight is reasonable or not directly affects the accuracy of the evaluation system.Here, we use analytic hierarchy process (AHP) to determine the weight of indicators. AHP was proposed by American scholar T.L. Saaty in 1977. The basic principle of AHP is to divide complex things into several ordered levels and construct a hierarchical structure model that can reflect the internal connections of the system. The model combines elements with common attributes into a group as a hierarchy of the structure model. We construct a "comparative judgment matrix". On the basis of the maximum eigenvalues of the matrix and its corresponding eigenvectors, and on the premise of passing the consistency test, the weight of the relative importance of each element is obtained by sorting at each level. Through the analysis of each level, the total weight ranking of the problem is obtained.

(1) Build hierarchical model.

The highest level refers to the purpose of the decision and the problem to be solved. The lowest level refers to alternatives when making decisions. The middle layer refers to the factors to be considered and the criteria for decision-making. For the two adjacent layers, the upper layer is called the target layer and the lower layer is called the factor layer.

(2) Construct the judgment matrix of two comparison The judgment matrix is a subjective judgment process. It is based on data data, expert opinions, and the experience of system analysts. After repeated research, it is determined. The judgment Matrix has the following properties:

$$
\begin{gathered}
b_{\mathrm{ij}}=1 / b_{\mathrm{ji}} \\
b_{\mathrm{ii}}=1 \\
b_{\mathrm{ij}}=b_{\mathrm{ik}} / b_{\mathrm{jk}} \quad(\mathrm{i}, \quad \mathrm{j}, \quad \mathrm{k}=1,2, \ldots . \mathrm{n})
\end{gathered}
$$


Ahp adopts the following 1-9 scale method to conduct quantitative evaluation of different situations.

Table 2. Scaling method for matrix element

\begin{tabular}{|c|c|}
\hline Scales & meaning \\
\hline 1 & As important \\
\hline 3 & A little important \\
\hline 5 & More important \\
\hline 7 & Highly important \\
\hline 9 & Extremely important \\
\hline $2,4,6,8$ & $\begin{array}{c}\text { Intermediate value of } \\
\text { two adjacent judgments }\end{array}$ \\
\hline
\end{tabular}

(3) Calculate the characteristic roots $\lambda_{\max }$ of the judgment matrix.

The summation method is used here. The normalization of each component of the eigenvector is the weight value of the corresponding element of the same level for a factor of the upper level.

(4) Matrix hierarchy sorting and consistency testing.

After the matrix $A$ is normalized, the matrix $Q$ is obtained

$$
A=\left[\begin{array}{cccc}
b_{\mathrm{ij}}=1 / \sum \mathrm{n}_{\mathrm{i}=1} & b_{\mathrm{ij}} \\
a 11 & a 12 & a 13 & a 14 \\
a 21 & a 22 & a 23 & a 24 \\
a 31 & a 32 & a 33 & a 34 \\
a 41 & a 42 & a 43 & a 44
\end{array}\right]
$$

We add the rows of the matrix $\mathrm{Q}$ to get a vector a.

Normalization of vector a, Make $w_{\mathrm{i}}=a_{\mathrm{i}} / \sum_{\mathrm{k}=1} a_{\mathrm{k}}$, Get the vector corresponding to the maximum eigenvalue $W=\left(W_{1}, W_{2}, W_{3} \ldots . ., W_{m}\right)$.

According to the above evaluation index system, the indexes of the main criterion layer, namely the first-level indexes, are:

$X=\left(X_{1}, X_{2}, X_{3}, X_{4}\right)$;

Sub-criteria layer indicators, namely secondary indicators are:

$$
\begin{aligned}
& X_{1}=\left(X_{11}, X_{12}, X_{13}\right), \\
& X_{2}=\left(X_{21}, X_{22}, X_{23}, X_{24}, X_{25}\right), \\
& X_{3}=\left(X_{31}, X_{32}, X_{33}, X_{34}, X_{35} X_{36}, X_{37}, X_{38}\right), \\
& X_{4}=\left(X_{41}, X_{42}, X_{43}, X_{44}\right),
\end{aligned}
$$

Let the weight of principal criterion layer B to target layer A be set as B1, B2, B3, B4, respectively, and $\sum$ ${ }_{i=1} B_{i}=1$, the corresponding weight matrix is $B=(B 1, B 2$, $\mathrm{B} 3, \mathrm{~B} 4)$. The weight of sub-criterion layer $\mathrm{C}$ to the main criterion layer B is $\mathrm{W} 1, \mathrm{~W} 2, \mathrm{~W} 3, \mathrm{~W} 4$.respectively, and $\sum{ }_{\mathrm{i}=1} \mathrm{~W}_{\mathrm{i}}=1$, The corresponding weight matrix is:

$$
\begin{aligned}
& W_{1}=\left(W_{11}, W_{12}, W_{13}\right), \\
& W_{2}=\left(W_{21}, W_{22}, W_{23}, W_{24}, W_{25}\right), \\
& W_{3}=\left(W_{31}, W_{32}, W_{33}, W_{34}, W_{35} W_{36}, W_{37},\right. \\
& \left.W_{38}\right),
\end{aligned}
$$

$W_{4}=\left(W_{41}, W_{42}, W_{43}, W_{44}\right)$,

If hierarchical ordering can be confirmed, consistency test is required. Where, the unique non-zero characteristic root of $n$-order uniform matrix is $n$; The maximum characteristic root of $n$-order positive reciprocal matrix $A$, if and only if $\lambda=n, A$ is an uniform matrix. Consistency indicators are defined as:

$$
C I=(\lambda-n) /(n-1)
$$

$\mathrm{CI}=0$, complete consistency; $\mathrm{CI}$ is close to 0 , with satisfactory consistency; The larger CI is, the more serious the inconsistency is. To measure the size of CI, random consistency index RI is introduced:

$$
R I=\left(C I_{1}+C I_{2}+\ldots+C I_{n}\right) / n
$$

In general, the higher the order of matrix, the higher the probability of consistent random deviation.

$$
C R=C I / R I
$$

If $\mathrm{CR}<0.1$, it is considered that the hierarchical ordering has a satisfactory consistency; otherwise, it is necessary to adjust and modify the judgment matrix of this level until the overall ordering of the hierarchy reaches a satisfactory consistency.

(5) The total weight conclusion is drawn.

\subsection{Flexibility assessment methodology}

(1) Determine the set of evaluation observations.

For the above-mentioned evaluation index system, the following comment set is adopted: $Y=(Y 1, Y 2, Y 3$, $Y 4, Y 5)=\{$ Strong, Secondary Strong, Medium, Weak, Poor\}.

(2) Establish fuzzy evaluation matrix.

Fuzzy evaluation matrix $A i(\mathrm{I}=1,2,3,4)$ is established for each evaluation index $\mathrm{Xi}$ in the main criteria layer. By considering the classification factor index Xij in layer $\mathrm{C}$, the degree of its subordination to the $\mathrm{t}$-th evaluation Yt is judged to be $r_{i j t}$, and Xi's fuzzy evaluation matrix Ri can be obtained. For example,

$$
R_{I}=\left[\begin{array}{lllll}
r_{111} & r_{112} & r_{113} & r_{114} & r_{115} \\
r_{121} & r_{122} & r_{123} & r_{124} & r_{125} \\
r_{131} & r_{132} & r_{133} & r_{134} & r_{135} \\
r_{141} & r_{142} & r_{143} & r_{144} & r_{145}
\end{array}\right]
$$

Now we can determine the fuzzy comprehensive discriminant set $\mathrm{Ai}$ of the first-level indicators. We use $\underline{\mathrm{A}} \mathrm{i}=\mathrm{Wi} \circ \mathrm{Ri}(\mathrm{I}=1,2,3,4)$ to obtain the comprehensive fuzzy discriminant set $\mathrm{Ai}=$ (ai1, ai2, ai3, ai4, ai5).

$$
\begin{aligned}
& \underline{A}_{1}=W_{1} \circ R_{l}=\left(a_{11}, a_{12}, a_{13}, a_{14}, a_{15}\right) \text {, } \\
& \underline{A}_{2}=W_{2} \circ R_{2}=\left(a_{21}, a_{22}, a_{23}, a_{24}, a_{25}\right) \text {, } \\
& \underline{A}_{3}=W_{3} \circ R_{3}=\left(a_{31}, a_{32}, a_{33}, a_{34}, a_{35}\right) \text {, } \\
& \underline{A}_{4}=W_{4} \circ R_{4}=\left(a_{41}, a_{42}, a_{43}, a_{44}, a_{45}\right) \text {, } \\
& \underline{A}=\left(\underline{A}_{1}, \underline{A}_{2}, \underline{A}_{3}, \underline{A}_{4}\right)^{T}
\end{aligned}
$$

Finally, the fuzzy evaluation matrix of the evaluation object is established: 


$$
\begin{aligned}
E & =B \circ \underline{A}=\left(e_{1}, e_{2}, e_{3}, e_{4}, e_{5}\right) \\
e_{i}^{\prime} & =e_{i} / \sum e_{i}(i=1,2,3,4,5) \\
E^{\prime} & =\left(e_{1}^{\prime}, e_{2}^{\prime}, e_{3}^{\prime}, e_{4}^{\prime}, e_{5}^{\prime}\right)
\end{aligned}
$$

ei 'corresponds to the previous comment element Yi respectively. From the perspective of probability, the probability in conformity of this ability and enterprise competitiveness is $\mathrm{e}_{i}$. According to the maximum membership principle in fuzzy mathematics, the core competitiveness of enterprises is evaluated.

\section{Case studies}

Company A is located in the East China region, where the proportion of sub-reimbursement costs for electricity auxiliary services is shown in Figure 1, and the proportion of each cost varies greatly. The company's total installed capacity of 600,000 kilowatts.

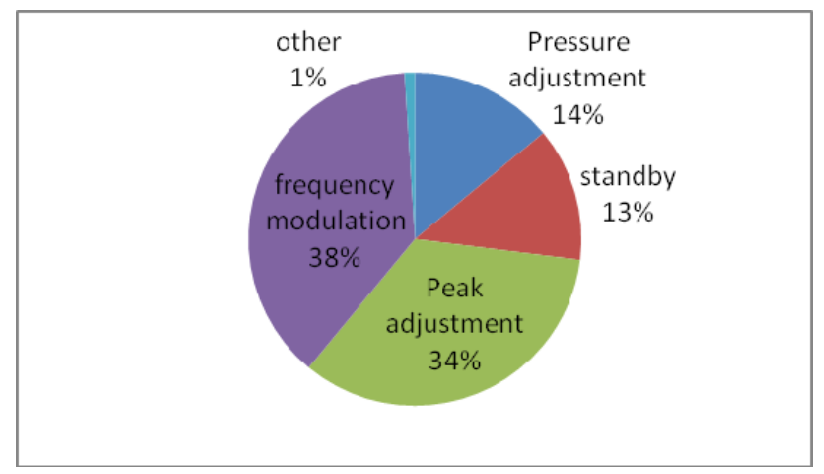

Fig. 1. Percentage of ancillary services

As can be seen from figure 1, the market demand in East China is more balanced, with more ancillary market services available to power producers and more flexibility requirements for generating units.

\subsection{Using the analytic hierarchy process (AHP) to weight the index}

By constructing judgment matrix and hierarchical single ordering, we complete the hierarchical total ordering and consistency test.

$W_{1}=\left(W_{11}, W_{12}, W_{13}\right)=(0.161,0.658,0.181)$

$W_{2}=\left(W_{21}, W_{22}, W_{23}, W_{24}, W_{25}\right)=(0.341,0.341,0.142,0.142,0$. 034);

$W_{3}=\left(W_{31}, W_{32}, W_{33}, W_{34,} W_{35} W_{36}, W_{37}, W_{38}\right)=(0.083,0.124,0$. $041,0.166,0.282,0.204,0.029,0.071)$;

$W_{4}=\left(W_{41}, W_{42}, W_{43}, W_{44}\right)=(0.241,0.402,0.153,0.204)$;

Table 3. Total hierarchical ordering.

\begin{tabular}{|c|c|c|c|c|c|c|c|c|c|}
\hline $\mathbf{c 1}$ & $\mathbf{c 2}$ & $\mathbf{c 3}$ & $\mathbf{c 4}$ & $\mathbf{c 5}$ & $\mathbf{c 6}$ & $\mathbf{c 7}$ & $\mathbf{c 8}$ & $\mathbf{c 9}$ & $\mathbf{c 1 0}$ \\
\hline 0.0235 & 0.096 & 0.026 & 0.088 & 0.088 & 0.036 & 0.036 & 0.0087 & 0.036 & 0.054 \\
\hline
\end{tabular}

\begin{tabular}{|c|c|c|c|c|c|c|c|c|c|}
\hline $\mathbf{c 1 1}$ & $\mathbf{c 1 2}$ & $\mathbf{c 1 3}$ & $\mathbf{c 1 4}$ & $\mathbf{c 1 5}$ & $\mathbf{c 1 6}$ & $\mathbf{c 1 7}$ & $\mathbf{c 1 8}$ & $\mathbf{c 1 9}$ & $\mathbf{c 2 0}$ \\
\hline 0.0178 & 0.0717 & 0.122 & 0.088 & 0.013 & 0.031 & 0.040 & 0.066 & 0.025 & 0.034 \\
\hline
\end{tabular}

Consistency test of c-level total ranking is shown in table 4.

Table 4. Total hierarchical ordering.

\begin{tabular}{|c|c|c|c|c|c|}
\hline \multirow{2}{*}{$\mathbf{C}$} & B1 & B2 & B3 & B4 & \multirow{2}{*}{ index } \\
\cline { 2 - 5 } & 0.146 & 0.257 & 0.432 & 0.165 & \\
\hline CI & 0.008 & 0.009 & 0.062 & 0.003 & 0.035 \\
\hline RI & 0.58 & 1.12 & 1.41 & 0.9 & 1.130 \\
\hline
\end{tabular}

$$
\begin{aligned}
& C I=\sum_{\mathrm{j}=1}^{\mathrm{m}_{1}} a_{j} C I_{j} \\
& R I=\sum_{\mathrm{j}=1}^{\mathrm{m}_{\mathrm{j}}} a_{j} R I_{j}
\end{aligned}
$$

$C R=C I / R I=0.035 / 1.130=0.031<0.1, \quad$ so $\quad$ the consistency of the overall ranking at level $\mathrm{C}$ is satisfactory.

\subsection{Fuzzy comprehensive evaluation and analysis}

Through questionnaire analysis and data processing, the fuzzy comprehensive analysis and evaluation of the core competitiveness of the independent power generation enterprise is conducted as follows:

(1) It is known that the weight vector of the subcriteria layer to the principal criteria layer is:

$W_{1}=\left(W_{11}, W_{12}, W_{13}\right)=(0.161,0.658,0.181)$;

$W_{2}=\left(W_{21}, W_{22}, W_{23}, W_{24}, W_{25}\right)=(0.341,0.341,0.142$,

$0.142,0.034)$;

$W_{3}=\left(W_{31}, W_{32}, W_{33}, W_{34}, W_{35} W_{36}, W_{37}, W_{38}\right)=(0.083,0.1$

24,0.041,0.166,0.282,0.204,0.029,0.071);

$W_{4}=\left(W_{41}, W_{42}, W_{43}, W_{44}\right)=(0.241,0.402,0.153,0.204)$;

(2) The weight vector of the principal criterion layer to the target layer is:

$B=\left(B_{1}, B_{2}, B_{3}, B_{4}\right)=(0.146,0.257,0.432,0.165)$.

(3) The following comment set is adopted: $Y=(Y 1$, $Y 2, Y 3, Y 4, Y 5)=\{$ Strong, Secondary Strong, Medium, Weak, Poor\}.

(4) Through expert investigation and analysis, the membership degree of indicator $\mathrm{Xij}$ to $\mathrm{Y}$ under factor index $\mathrm{Xi}$ of $\mathrm{C}$-layer is sorted out, and the fuzzy evaluation matrix $\mathrm{Ri}$ of $\mathrm{Xi}$ is obtained.

(5) The membership degree of each index of the main criterion layer to $\mathrm{Y}$ is confirmed, and the fuzzy matrix A $(\mathrm{X})$ is obtained.

$$
\begin{aligned}
& \underline{A}_{1}=W_{1} \circ R_{l}=(0.318,0.482,0.143,0.012,0.005), \\
& \underline{A}_{2}=W_{2} \circ R_{2}=(0.448,0.228,0.113,0.119,0.092), \\
& \underline{A}_{3}=W_{3} \circ R_{3}=(0.518,0.318,0.132,0.022,0.01), \\
& \underline{A}_{4}=W_{4} \circ R_{4}=(0.214,0.373,0.205,0.123,0.014), \\
& \underline{A}=\left(\underline{A}_{1}, \underline{A}_{2}, \underline{A}_{3}, \underline{A}_{4}\right)^{T}
\end{aligned}
$$

(6) The degree of subordination of the comment set $\mathrm{Y}$ to the total factor competitiveness of power generation enterprises

$E=B \circ A=(0.432,0.318,0.165,0.022,0.092)$.

Normalization is carried out:

$\sum_{i=1}^{5} e_{i}=1.029$,

$e_{i}{ }^{\prime}=e_{i} / \sum e_{i}(i=1,2,3,4,5)$ 
$E^{\prime}=\left(e_{1}^{\prime}, e_{2}^{\prime}, e_{3}^{\prime}, e_{4}^{\prime}, e_{5}^{\prime}\right)=$

$(0.420,0.309,0.160,0.021,0.089)$

Where, $e_{i}$ respectively corresponds to the previous comment set element $Y i$.

(7) From the above calculation, it can be seen that the degree of flexibility is $43.2 \%$, and the general degree of flexibility is $31.8 \%$. According to the maximum membership principle, we can judge the flexibility of the power generation company is strong.

\section{Conclusion}

Flexibility evaluation of power generation enterprises is a concept with wide coverage and complex content. Particularly in the context of the market for ancillary services, the need for flexibility varies from region to region, requiring a one-zone evaluation to be tailored to the needs of the market.

This paper analyzes the flexibility of power generation enterprises from four aspects: regional policy, market demand, flexibility index and cost. The evaluation of flexibility index is constructed, and it is hoped that it will have a guiding significance for the flexibility transformation of power generation enterprises in the future.

\section{Acknowledgement}

The auther gratefully acknowledge the contributions of the Science and technology project of China Huadian Corporation Ltd. ( CHDKJ18-01-73)

\section{References}

1. Chen Huang, Guangfu Wu, Wei Zheng. Power System Power System Engineering [J]. 3, (2018) : $14-20$

2. Jia Hu, Linxian Hu. Power System Protection and Control [J]. 19, (2013):13-16

3. Yongxiu He, Qian Chen, Yunzhi Fei, ChengRan Fu. Power System Technology [J]. 9, (2018):2916-2922

4. Ji Zhang, Lizi Zhang, Liang Yu. Proceedings of the CSEE [J]. 6, (2006) :124-128

5. Jianzhen Han, Shihua Cao, Xudong Liu, Jingang Zhang, Dong Li.HEBEI Electric Power $[\mathrm{J}]$. 3, (2019):56-59 